\title{
Balkanologie
}

Balkanologie Revue d'études pluridisciplinaires

Vol. $15 n^{\circ} 1 \mid 2020$

Mémoires performatives : faire des passés et des présents

\section{Note sur le tourisme en Albanie pendant la période zogienne (1925-1939)}

Note about tourism in Albania during Zog's period (1925-1939)

\section{Pierre Vilvens}

\section{(2) OpenEdition}

\section{Journals}

Édition électronique

URL : https://journals.openedition.org/balkanologie/2535

DOI : 10.4000/balkanologie.2535

ISSN : 1965-0582

Éditeur

Association française d'études sur les Balkans (Afebalk)

Référence électronique

Pierre Vilvens, « Note sur le tourisme en Albanie pendant la période zogienne (1925-1939)»,

Balkanologie [En ligne], Vol. 15 n$^{\circ} 1$ | 2020, mis en ligne le 01 juin 2020, consulté le 05 août 2021. URL http://journals.openedition.org/balkanologie/2535; DOI : https://doi.org/10.4000/balkanologie.2535

Ce document a été généré automatiquement le 5 août 2021

(c) Tous droits réservés 


\title{
Note sur le tourisme en Albanie pendant la période zogienne (1925-1939)
}

\author{
Note about tourism in Albania during Zog's period (1925-1939)
}

\section{Pierre Vilvens}

La période zogienne (présidentielle et monarchique) est une ère de transformation de l'Albanie à différents niveaux, notamment économique. Cette volonté de modernisation est néanmoins marquée par des concessions envers les grandes puissances et la soumission envers l'Italie voisine. Les différents accords de 1925 prévoyant la création d'une banque nationale et d'une société de développement économique, sous contrôle italien, symbolisent la difficulté de l'Albanie à développer son économie. Cette mainmise a été renforcée par le déficit de la balance commerciale après la crise de 1929, la difficulté de remboursement du prêt italien et les chantages financiers de Rome au début des années 1930, l'arrêt des exportations vers l'Italie et l'échec des relations commerciales entre la Grèce et l'Albanie ${ }^{1}$. Si les secteurs agricole et pétrolier sont souvent évoqués ${ }^{2}$ pour cette période, un secteur alors naissant est souvent oublié : le tourisme. À l'instar de ses voisins grec et yougoslave, plus précoces dans le développement touristique, l'Albanie a cherché à développer cette branche de l'économie pour obtenir des rentrées financières (on passe de 3736 touristes en 1937 à 5936 en 1938, et à des recettes de 125708 à 339241 francs-or) $)^{3}$. L'historiographie aborde le tourisme à la période communiste et post-communiste, mais il n'existe pas de travail de synthèse sur la période antérieure ${ }^{4}$. L'entre-deux-guerres est présente dans deux types de travaux. Il s'agit de biographies de personnalités qui ont œuvré politiquement (Zog, la princesse Maxhide, sœur de ce dernier, chargée de promouvoir le tourisme ${ }^{5}$ ) ou culturellement (la mise en valeur du pays par les découvertes archéologiques du Français Léon Rey ${ }^{6}$ ) à faire du pays un lieu de villégiature. Le second type, plus étudié et plus récent, est consacré aux récits de voyageurs occidentaux et à l'image donnée par ceux-ci'. Si les conditions d'accueil ne sont souvent qu'un détail parmi d'autres, elles jouent leur rôle dans la vision du pays. Le déficit historiographique 
sur le sujet semble, depuis quelques années, commencer à être comblé, notamment par la mise en valeur de certaines sources (infra). La promotion de l'Albanie comme destination bon marché dans un cadre préservé depuis quelques années et la volonté d'intégrer l'Union européenne ont sans doute été d'importants stimulants à cette découverte de l'histoire du tourisme en Albanie. Nous proposons ici une première description des sources disponibles.

2 Les sources inédites les plus importantes sont les archives du ministère des Affaires étrangères et de l'Europe d'Albanie ${ }^{8}$. Les principaux documents permettant de retracer le début de l'activité touristique ont été numérisés et mis en ligne en 2018 par le ministère. Ce dernier retrace déjà sur son site internet la politique de développement touristique pour les années 1925-1928. Il s'agit d'un corpus de sources comprenant essentiellement des lettres émanant de diplomates et consuls, ainsi que des ministres Husejn (1882-1938) et Iliaz (1882-1932) Vrioni. Les documents sont écrits en plusieurs langues, en albanais, français et anglais essentiellement. Le corpus couvre la période du 12 novembre 1926 au 19 janvier 1928. Plusieurs archives font doublon, car il s'agit de traductions. Le document le plus intéressant, à plus d'un titre, est sans doute le premier, une lettre de Malik Libohova (né en 1896), alors chargé d'affaires ad interim à Paris. Elle met en évidence le souhait de créer une agence officielle du tourisme pour créer de nouvelles richesses. Dans ce but, le diplomate prend contact avec le dénommé Larcher, directeur des agences de la Compagnie française (de tourisme ?) et Raoul de Brie (1877-1950), administrateur-délégué des voyages « Étoile ». Libohova envisage que l'agence - laquelle pourrait devenir un office centralisé composé des acteurs du tourisme - puisse avoir des correspondants en France et à l'étranger. Ces derniers seraient chargés de l'accueil des touristes en Albanie, de la propagande et de la préparation des voyages depuis l'étranger. Le diplomate imagine d'ailleurs que «l'organisation extérieure [sic]» (publicité et transport des touristes) soit réalisée par l'agence «Étoile »'. Cette agence de voyage n'a pas été choisie au hasard. Depuis 1924, elle cherche à devenir le correspondant des hôteliers d'Europe centrale et orientale ${ }^{10}$. De plus, son adresse parisienne - le 6 avenue Kléber - sera le siège de l'office du tourisme albanais ${ }^{11}$. Les deux lettres destinées au ministre des Affaires étrangères Vrioni mettent en évidence le rôle de Léon Rey en tant qu'intermédiaire entre les autorités albanaises et les professionnels du tourisme ${ }^{12}$, comme le remarque Etleva Nallbani ${ }^{13}$. Le reste des documents, datés du 26 décembre 1926 au 19 janvier 1928, concerne exclusivement le développement du tourisme automobile et la création d'un touring club albanais (KTAM, 1927) ${ }^{14}$ sur la base des modèles occidentaux, en particulier anglais. En 1927, des contacts sont établis entre l'Automobile Association de Londres et le gouvernement albanais pour favoriser le tourisme automobile. L'association enverra deux de ses membres, Arthur Dunscombe Allen (1882-1969) et le colonel Etherton $(1879)^{15}$, pour juger de la situation (dont ils tireront un ouvrage) ${ }^{16}$. Ces documents, qui renseignent sur les buts et actions du club, confirment les informations données par l'officier britannique en poste en Albanie, Dayrell Oakley-Hill (1898-1985) ${ }^{17}$, ancien conseiller de Zog. La consultation de documents postérieurs (mais pas encore mis en ligne) sera certainement nécessaire pour mesurer l'importance de ce club, qui n'est devenu membre de l'Association internationale des Automobile Clubs reconnus qu'en octobre $1933^{18}$. S'agissant des années 1930 , le ministère a déjà réalisé des inventaires (mis en ligne) pour les années $1937^{19}, 1938^{20}$ et $1939^{21}$. On y trouve des documents sur la politique touristique du gouvernement (correspondance sur le rôle des représentations à l'étranger, l'action du KTAM, les contacts avec les agences de voyages étrangères) et 
sur le Enti Kombëtar i Turizmit. Créé en 1936, il s'agit d'un bureau national pour développer le tourisme, via la facilitation de la délivrance de passeports, la modernisation des hôtels et la propagande touristique (films, cartes... ${ }^{22}$. La consultation d'archives consulaires des principales puissances ${ }^{23}$ sera sans doute intéressante. Par contre, les archives diplomatiques éditées ne semblent pas ou peu utiles pour notre problématique. Pour les sources iconographiques, il existe un film de propagande touristique à destination du public anglo-saxon. The March of Times, tourné en 1936 par une compagnie américaine, s'est détourné de son but originel et a donné une image biaisée du régime ${ }^{24}$. Il semble par contre n'y avoir aucune affiche de propagande touristique.

3 Les sources éditées sont évidemment plus aisément consultables. On en compte cinq types. Il s'agit, en premier lieu, des guides touristiques, qui doivent inciter (ou non) à partir. L'Albanie semble avoir été négligée par les guides occidentaux. Le Baedeker ne lui consacre qu'un guide en 1929, en allemand seulement et en lien avec la Grèce et la Yougoslavie ${ }^{25}$. Il fournit néanmoins de précieuses indications et décrit sans détour les conditions de villégiature, en particulier la qualité des logements et des restaurants ${ }^{26}$. Le cas français ${ }^{27}$ est plus ou moins contemporain car le premier guide sur le pays, dû à Rey, date de $1930^{28}$. Il comprend des conseils, un aperçu sur différents points du pays, une vingtaine d'itinéraires, le plan des trois principales villes (Tirana, Shköder et Korçë) et une carte des voies d'accès. Jacques Ancel en juge les représentations géographiques trop sommaires ${ }^{29}$. En 1972, il est jugé dépassé «mais encore utile pour le visiteur non averti $»^{30}$. Pour le cas anglais, il faut mentionner le Foreign guide de l'Automobile Association, qui donne les conditions d'accès (pour l'automobile exclusivement) et une liste très précise des établissements (aux noms similaires), mais une information succincte (système de cotation par étoile) sur la qualité de ceux-ci ${ }^{31}$. Le guide suisse Europa touring (trilingue) donne des informations similaires et renvoie au Baedeker, au Rey et à l'automobile-club albanais ${ }^{32}$.

4 Le deuxième type concerne les récits de voyages, essentiellement d'aventuriers, publicistes et voyageurs anglais ${ }^{33}$. Les ouvrages en allemand sont davantage des études scientifiques et ne semblent pas correspondre à notre corpus ${ }^{34}$. Pour la plupart, il s'agit d'histoires anecdotiques et le lyrisme ou l'exagération sont les risques classiques de tels récits. Le cas le plus soumis à caution est celui de Joseph Swire (1903-1978) qui voyagea à plusieurs reprises dans les Balkans. Après avoir donné une image favorable de Zog dans son premier livre, il fut payé par le gouvernement italien pour fournir des articles favorables à la politique albanaise de l'Italie dans la presse anglo-saxonne au début des années $1930^{35}$. Si l'image de l'Albanie reflétée par ce genre littéraire a déjà été étudiée ${ }^{36}$, les récits de voyage ont l'avantage de donner plus de sens à la réalité que certains guides. On peut citer le récit de l'ethnologue et voyageuse Jacqueline Bénézech (1903-2004), qui est un des rares documents français réellement intéressants ${ }^{37}$. Lors d'un voyage avec son époux artiste dans les Balkans, elle passe en Albanie (10-18 janvier 1931) et présente le Grand Hotel de Shköder, que Rey leur avait recommandé, comme une pauvre bâtisse dans une cour de terre battue, sans eau courante $^{38}$. En plus des lieux, elle donne des indications sur la population, le décor et certaines personnalités. Par exemple, elle dresse le portrait de l'agent consulaire Spendjopoulo ${ }^{39}$. Or ce dernier est le représentant de l'agence «Étoile $»^{40}$. De manière générale, les récits donnent une image plutôt négative de la plupart des établissements : délabrement, qualité des services médiocres. Ils confirment le rôle de plus en plus important de l'Italie dans le secteur touristique, qui devient officiel au 
moment de l'invasion italienne (avril 1939), avec le développement de l'infrastructure hôtelière par l'ENITEA ${ }^{41}$, l'équivalant albanais de l'agence nationale du tourisme d'Italie. Cette influence, qui se traduit dans la cuisine et le personnel, ne se rencontre que dans les meilleurs établissements, qui sont le Continental Hotel à Durrës ${ }^{42}$, le Continental, l'International et le Dajti Hotel ${ }^{43}$ à Tirana. Si le récit francophone le plus précis sur les hôtels est celui du couple Bénézech, qui décrit de nombreux établissements, on peut y ajouter le reportage de René Vanlande $(1886)^{44}$, critique sur le protectorat italien en Albanie. Il donne d'intéressants renseignements sur la douane, les modalités de change, l'hôtel de Durrës et ceux de Tirana (que l'apport italien n'aurait guère améliorés), la difficulté de photographier (il faut une autorisation du ministère de l'Intérieur) et les drogmans ${ }^{45}$. Le texte anglophone le plus complet est celui de l'Américain John Irvin Beggs McCulloch (1903-1988) ${ }^{46}$ qui donne des informations très précises sur le Continental Hotel de Tirana ${ }^{47}$.

Certains établissements semblent avoir perduré sous la période communiste (il n'y a des hôtels que dans les grandes villes) ${ }^{48}$, voire au-delà (tel l'hôtel thermal de Llixhat, toujours en activité $)^{49}$. En lien avec les récits de voyages, on peut ajouter les mémoires et « récits de vie » (biographie, journal) de contemporains. Pour la France, on ne peut citer que l'écrivain Valéry Larbaud (1881-1957), qui voyage en Albanie en 1935 à l'invitation du diplomate Marcel Ray (1878-1951). Dans son journal, il exprimera sa sympathie pour ce petit pays et son peuple ${ }^{50}$. Pour le cas anglo-saxon, j'ai évoqué les mémoires de Oakley-Hill. Ces dernières ont été éditées par Bejtullah D. Destani. Elles rapportent une autre entreprise évoquée par Zog dans une interview de 1935. Ce dernier avait imaginé de multiplier les routes et chalets touristiques dans les collines ${ }^{51}$. Selon l'officier britannique, le gouvernement n'a pas eu le temps ni les moyens de concrétiser cette entreprise ${ }^{52}$. Cette action est plus détaillée par son compatriote Swire, qui envisageait la création d'une agence anglaise en Albanie, la création d'un office du tourisme, la publication d'un périodique et la mise en place de normes hôtelières. Hormis le deuxième élément, rien ne semble avoir été réalisé ${ }^{53}$.

6 L'avant-dernier type de sources éditées est la presse. La consultation de la presse généraliste, notamment allemande (via ZEFYS [Zeintungsinformationssystem]), anglaise (via Britishnewspaperarchives), française (via Gallica), suisse (via enewspaperarchives) est nécessaire pour trouver des mentions de ce qui stimule les gens à partir (voyages, conférences...). La presse permet de donner des renseignements sur les croisières organisées, entre autres, par l'association Guillaume Budés4, que ce soit sur leur but (visite de vestiges archéologiques) ou sur leur organisation (coût, escales...). Ce travail long et patient permet de mettre en évidence l'apport de certaines personnalités (comme l'organisation d'une croisière par le diplomate francophile Stavro Stavri $[1882-1954]^{55}$ en 1933$)^{56}$ dans la promotion de l'Albanie. La presse spécialisée doit également être parcourue, notamment la presse sur le tourisme ( $L a$ Revue du Touring-club de France) et la presse consacrée à la région balkanique. Je pense en particulier à la revue Les Balkans ${ }^{57}$ et à l'article du diplomate Skënder Konitza sur le tourisme en Albanie, qui met en évidence les nombreuses activités existantes (chasse, pêche, thermalisme) et futures ${ }^{58}$. La presse concernant les moyens de transport ${ }^{59}$ ne doit pas être obligatoirement consultée, les guides fournissant déjà des informations. Elle offre néanmoins des détails sur les lignes d'aviation ou de bateaux, ainsi que sur la réalisation ou non de certains projets (projet du syndicat international anglo-italien Balkans Railways d'une ligne Durazzo-Salonique ${ }^{60}$. 
7 Enfin, dans les sources diverses, une attention doit être portée aux documents concernant la participation à des manifestations internationales, telles que les expositions internationales, ou aux congrès internationaux de tourisme. À notre connaissance, c'est seulement lors de l'Exposition de New York (1939) que l'Albanie a mis l'accent sur le tourisme, en installant un bureau d'accueil et de renseignements ${ }^{61}$.

\section{NOTES}

1. Castellan, Georges, Histoire de l'Albanie et des Albanais, Crozon, Armeline, 2002, p. 77-78.

2. Ibid., p. 77-80.

3. STAVRE Benita, Shqipëra dhe Shqiptarët Gjatë viteve 1921-1939 në shkrimet e autorëve anglezë [L'Albanie et les Albanais dans les années 1921-1939 dans les écrits d'auteurs anglais], Thèse de doctorat en langues, inédit, Université de Tirana, 2013, p. 171.

4. BAKIU Vjollca, BAUM Tom, "Tourism in Albania, Competing in the Mediterranean Region", Anatolia, vol. 10, $\mathrm{n}^{\circ}$ 1, 1999, p. 5-14; BONJA Alma, Le développement du tourisme international en Albanie, Mémoire de master, Igeat-Université libre de Bruxelles, année académique 2009-2010 ; ELSIE Robert, "Tourism ", dans Historical Dictionary of Albania, $2^{\mathrm{e}}$ édition, Manham, Toronto, Plymouth, The Scarecrow Press, 2010 (Historical Dictionaries of Europe, n ${ }^{\circ} 75$ ), p. 450 ; LICAJ Brunilda, Molla Armanda, "'Peeping' Tourist: A Case study of the State Tourist AgencyAlbTourist ", présentation donnée à The 9th International Association for History of Transport Traffic and Mobility, (TM2), Berlin (6-9 octobre 2011), en ligne: https://www.academia.edu/17766878/ _PEEPING_TOURIST_A_CASE_STUDY_OF_THE_UNIQUE_STATE_TOURIST_AGENCY_IN_ALBANIA_ALBTURIST (consulté en juillet 2019).

TOMES James, King Zog. Self-made monarch of Albania, Stroud, Sutton, 2003, p. 111.

5. ELSIE Robert, «Zogolli, Maxhide (1904 ?-1969)», dans A Biographical Dictionary of Albanian History, Londres, New York, I.B. Tauris, 2012, p. 504-505.

6. NALLBANI Etleva, «Léon Rey, pionier $i$ arkeologjisë franceze në Shqipëri » [Léon Rey, pionnier de l'archéologie française en Albanie], Iliria , vol. 12, 2003, p. 31-46.

7. BÉNÉZECH-MONOD-HERZEN Jacqueline, Carnets de voyages en Albanie (1931-1938), édition critique de Josiane Barbier, Paris, L'Harmattan, 2018 ; HEMMING Andreas, « German-speaking Travel Writers in interwar Albania ", dans Andreas Hemming, Gentiana Kera, Enriketa Pandelejmoni (dir.), Albania. Family, Society and Culture in the 20th Century, Münster-Viennes, Lit, 2012, p. 115-131; STAVRE Benita, Shqipëra dhe Shqiptarët, op. cit., p. 51, 171, 182, 274.

8. «Roli i legatave dhe konsullatave shqiptare jashtë vendit në zhvillimin e turizmit të huaj në Shqipëri në vitet '20-të të shek.XX» [Le rôle des légations et consulats dans le développement du tourisme étranger en Albanie dans les années 1920], Republika e Shqipërisë, Ministère de l'Europe et des Affaires étrangères, 2018, en ligne: https://punetejashtme.gov.al/en/roli-i-legatave-dhekonsullatave-shqiptare-jashte-vendit-ne-zhvillimin-e-turizmit-te-huaj-ne-shqiperi-ne-vitet-20te-te-shek-xx/ (consulté en juillet 2019).

9. Archives du ministère des Affaires étrangères et de l'Europe d'Albanie, Tirana, Lettre (en français) de Malik Libohova, ministre d'Albanie à Paris, à Husejn Vrioni, ministre des Affaires étrangères, Paris, 12 novembre 1926. 
10. France-Europe orientale. Bulletin officiel du Comité national d'expansion économique dans l'Europe orientale, 17 octobre 1924, n. p.

11. La Revue du Touring-club de France, mai 1931, p. 152.

12. Archives du ministère des Affaires étrangères et de l'Europe d'Albanie, Tirana, Lettre $\mathrm{n}^{\circ} 4041 / \mathrm{J}$, Lettre (en français) de Malik Libohova, ministre d'Albanie à Paris, à Husejn Vrioni, ministre des Affaires étrangères, Paris, 12 novembre 1926.

13. NALLBANI, « Léon Rey », art. cit., p. 31-46.

14. Archives du ministère des Affaires étrangères et de l'Europe d'Albanie, Tirana, Lettre de Léon Rey à S.E. Iliaz Vrioni, 28 décembre 1926, p. 1.

15. Archives du ministère albanais des Affaires étrangères et européennes, Lettre $n^{\circ} 20$, Lettre (en albanais) d'un fonctionnaire du ministère de l'Intérieur au ministre des Affaires étrangères, Tirana, 20 mars 1927.

16. ETHERTON Percy Thomas, DUnSCOMBE ALLEN Arthur, Through Europe and the Balkans. The Record of a Motor Tour, Londres, Cassell, 1928.

17. OAKLEY-HILL Dayrell R., An Englishman in Albania. Memoirs of British Officer, 1929-1955, édité par Bejtullah Destani, Londres, I.B. Tauris, 2004, p. 51.

18. L'Auto-Vélo, 17 octobre 1933, p. 2, col. 5.

19. Ministria për europën dhe punët e jashtme arkivi historiko- diplomatik. Inventari i Dokumentacionit $i$ Vitit 1937 MPJ, s.l. (Tirana (?), s.n., juillet 2018, p. 41, 43, 44.

20. Ministria për europën dhe punët e jashtme arkivi historiko- diolomatik. Inventari i Dokumentacionit $i$ Vitit 1938 MPJ, s.l. (Tirana (?), s.n., juillet 2018, p. 12, 30, 39, 40, 45, 47, 51.

21. Ministria për europën dhe punët e jashtme arkivi historiko- diolomatik. Inventari i Dokumentacionit $i$ Vitit 1939 MPJ, s.l. (Tirana (?), s.n., juillet 2018, p. 2, 13, 20.

22. STAVRE, Shqipëra dhe Shqiptarët, op. cit., p. 171.

23. Pour retrouver les consulats, la consultation de l'Almanach de Gotha sera nécessaire.

24. Foreign Relations of the United States. Diplomatic Papers 1936, vol. II, Europe, Washington, ÉtatsUnis, Government Printing Office, 1954, p.1-4. Le film montrait une scène dans laquelle Zog invitait des touristes étrangers à participer à une course de voitures la nuit. Une autre séquence du film annonçait le remplacement du major-général Sir Jocelyn Percy (1871-1952), inspecteurgénéral de la gendarmerie (1927-1938), au profit d'officiers italiens.

25. BAEDEKER Karl (dir..), Baedeker's Dalmatien und die Adria [Baedeker's. Dalmatia and the Adriatic], trad. anglaise Michael Wild, Plymouth, The Red Scar Press, 2018 [1929].

26. BAEDEKER, Baedeker's Dalmatien und die Adria, op. cit., p. 298.

27. Les guides bleus d'Hachette ne s'y sont jamais intéressés et Michelin ne s'est intéressé au pays que dans les années 1990.

28. REY Léon, Guide de l'Albanie. Avec une carte des voies d'accès, une carte routière et les plans de Scutari, Tirana et Kortcha, Paris, Office du tourisme en Albanie, 1930.

29. colin Elicio (dir.), Bibliographie géographique internationale, 1930 ( $\mathrm{XL}^{e}$ Bibliographie annuelle), Paris, Armand Colin, 1931, p. 280.

30. zAKHOS Emmanuel, Albanie, Paris, Seuil, 1972, p. 100.

31. Foreign Touring Guide, Automobile Association (Great Britain), Londres, Automobile Association, 1931, p. 402-406.

32. Europa touring. Guide automobile d'Europe. Automobilführer von Europa. Motoring guide of Europe, Berne, Hallwag, 1937, p. LXIII.

33. MINTA Stephen, « Albania », dans Jennifer Speake (dir.), Litterature of Travel and Exploration. An Encyclopedia, vol. I, A-F, New York, Londres, Fitzroy Dearborn, 2003, p.9-11; Young Antonia, HODGSON John, YOUNG Nigel, World bibliographical series, vol. 64, Albania, Oxford, Clio Presses, 1997 [1988], p. 31. Il s'agit de: The Unambitious Journey, Londres, Chapman and Hall, 1935, par l'écrivaine Theodora Benson ; Two Vagabonds in Albania, New York, Dodd, Mead and Co et Londres, 
John Lane, 1927, par les voyageurs, écrivains et artistes Jan et Cora Gordon; le publiciste et ami de Graham Greene Ronald Matthews avec Sons of the Eagle, Wanderings in Albania, Londres, Methuen, 1937 ; le journaliste Joseph Swire Albania. The Rise of a Kingdom, Londres, William and Norgate, 1929 et King Zog's Albania, Londres, Hale, 1937. Le seul auteur américain est John Irvin Beggs McCulloch avec Drums in the Balkan Night, New York, G.P. Putnam's Sons, 1936.

34. HEMMING, « German-speaking Travel Writers », art. cit., p. 115-131.

35. TROEBST Stefan, « Ein britischer Journalist im Sold des faschistischen Italien. Joseph Swire als Korrespondent in Albanien in den Jahren 1930/31 ", Themenportal Europaïsche Geschichte, 2007, en ligne : <www.europa.clio-online.de/essay/id/fdae-1434> (consulté en novembre 2019).

36. STAVRE, Shqipëria dhe Shqiptarët, op. cit., p. 51, 171, 182, 274.

37. BÉNÉZECH-MONOD-HERZEN, Carnets de voyages en Albanie, op. cit.

38. Ibid., p. 13, 22. Ils y reviennent entre le 16 août 1938 et le 15 janvier 1939.

39. Ibid., p. 25-31. Dans son ouvrage, Jacqueline Bénézech l'écrit Schpendopoulos. Valéry Larbaud l'évoque aussi dans son journal et sa correspondance.

40. Archives du ministère albanais des Affaires étrangères et de l'Europe, Tirana, Lettre de Léon Rey à S.E. Iliaz Vrioni, Paris, 28 décembre 1926, p. 2.

41. SYRJAMAA Taina, Visitez l'Italie. Italian state tourist propaganda abroad, 1919-1943. Administrative structure and Practical realization, Turku, Turum Yliopisto, 1997 (Annales Universitatis Turkuensis, ser. B. Humaniora, 217), p. 180, 261, 281.

42. MERCIER Armand, «L'Aigle Noir (III ${ }^{\mathrm{e}}$ partie) », La Revue de France, $\mathrm{n}^{\circ}$ 17, 1937, p. 279-311.

43. TOMES, King Zog, op. cit., p. 111.

44. Archives départementales du Pas-de-Calais, Dainville, Matricules, classe 1906, matricule 3163, matricule de René Vanlande, 1909-1937 ; Annuaire général des lettres 1933, Paris, s.n., 1933, p. 1183. René Vanlande, officier et publiciste français, collaborateur de L'Écho de Paris et de diverses publications coloniales, titulaire de nombreuses décorations françaises (dont la Légion d'honneur) et étrangères, cet ancien officier est l'auteur de nombreux ouvrages sur des régions qu'il a fréquentées. On peut citer la Prusse et la Lituanie avec le général Niessel ou le Maroc sous les ordres de Lyautey.

45. vanLande René, En Albanie sous l'œil de Mussolini, Paris, Peyronnet, 1933, p. 19, 20, 23, 24, 27, 75-78, 102-104, 111-113. Bien que publié en 1933, l'ouvrage semble se situer entre 1928 (Zog devient roi) et 1930, car l'auteur cite Léon Rey préparant un guide. Or, ce dernier est paru en 1930. De plus, un représentant de Baedeker serait déjà venu, ce qui est une référence au guide de 1929.

46. Who Was Who in America, with world notables, vol. VIII, 1982-1985, Chicago, Marquis-Who's Who, 1985, p. 185. John Irvin Beggs McCulloch (1908-1983), petit-fils d'un richissime homme d'affaires de l'Est des États-Unis. Auteur prolifique, cet ancien étudiant des universités de Yale et Stanford s'intéressa à la politique internationale. Il fut le rédacteur en chef de Inter-Americas (1939-1945) et participa à la Seconde Guerre mondiale dans l'oss.

47. MCCUlloch, Drums in the Balkan Night, op. cit., p. 139, 166.

48. Europa Touring, Berne, Hallwag, 1965, p. 765.

49. GLOYER Gillian, Albania, $3^{\mathrm{e}}$ édition, Chalfont St Peter, Brandt travel Guides, 2008, p. 101.

50. LARBAUD Valéry, Journal, préfacé et annoté par Paule Moron, Paris, Gallimard, 2009 [1954], p. 1483 ; WEISSMAN Frida, L'exotisme de Valéry Larbaud, Paris, Librairie A.G. Nizet, 1966, p. 228.

51. BISCARRE Alex, «Impressions d'Albanie. Sur la route de la capitale de Zog $\mathrm{I}^{\mathrm{er}}$ », Le Matin, 17 juin 1935, p. 2, col. 2-3.

52. OAKLEY-HILL, An Englishman in Albania, op. cit., p. 51.

53. SWIRE, King Zog's Albania, op. cit., p. 219-220. Selon Swire, ces projets n'ont pas abouti suite à une mésentente non avec Zog, mais avec les autorités, en particulier avec le ministre de 
l'Intérieur Musa Juka (1885-1955). Pour ce dernier, l'action gouvernementale devait se limiter au passeport pour groupes touristiques.

54. BOMPAIRE Jacques, «75 $75^{\mathrm{e}}$ anniversaire », Bulletin de l'association Guillaume Budé, 3, octobre 1994 , p. 264-266. L'association Guillaume Budé (toujours en activité) est une société savante fondée en 1917 pour promouvoir, défendre et mettre en valeur l'héritage gréco-romain (via des conférences, voyages...) et la science française dans le domaine classique. Deux ans plus tard, les fondateurs de la société créent la société d'éditions «Les Belles lettres ", destinée à la publication d'auteurs classiques. Sur les voyages de l'association en Grèce, voir cHÈzE Mathilde, « Les Français en Grèce: du tourisme de lettrés au tourisme de masse (années 1930-années 1990)», Histoire@Politique.Politique, culture, société, vol. 28, nº 1, 2016, p. 126-144.

55. Archives nationales, Paris, Dossier des titulaires de la Légion d'honneur, Dossier $n^{\circ} 60.920$, Dossier de Stavro Stavri, 1954-1955. Stavro Stravi, diplomate albanais. Il fut successivement ministre à Belgrade et à Athènes, délégué à la SDN et ministre d'Albanie à Paris. Il est l'auteur de nombreuses publications sur son pays. Stavri participa à la Première Guerre mondiale en tant que volontaire dans l'armée française et fut résistant pendant la Seconde. Le seul ouvrage traitant (en partie) de Stavri est SHEHI Zyhdi, Inxhinieri Jani (Xhuf) Koljaka. Diplomati Stavro Stavri [L'ingénieur Jani (Xhuf) Koljaka. Le diplomate Stavro Stavri.], Durrës, DDS, 2014.

56. Le Temps, 24 mai 1933, p. 5, col. 4.

57. Il s'agit d'une revue politique trimestrielle publiée à Athènes à partir de 1930. Rédigée en français, elle est un «organe de rapprochement interbalkanique ». Publiée avec l'appui de la Dotation Carnegie pour la paix internationale, la revue est patronnée par le Premier ministre grec Papanastasiou. On y trouve des articles sur tous les aspects des questions balkaniques ainsi que des textes authentiques et intégraux des documents officiels de la Conférence balkanique. KERSOPOULOS Jean G., « Bibliographie juridique sur la Grèce », Revue hellénique de droit international, vol. 27, 1964, p. 163-172 ; KIтSIKIs Dimitri, «Les projets d'entente balkanique, 1930-1934 », Revue historique, $\mathrm{n}^{\circ} 241$, juin 1969, p. 115-140.

58. KONITZA Skënder, «Le tourisme en Albanie », Les Balkans, septembre-octobre 1933, p. 607-614.

59. Nous pensons, entre autres, à: L'Aéronautique. Revue mensuelle illustrée, Les Ailes. Journal hebdomadaire de la locomotion aérienne, The Nautical Gazette, Revue générale des chemins de fer.

60. Revue générale des chemins de fer, vol. 48, n 3, septembre 1929, p. 269.

61. MONAGHAM Frank, Official Guide Book. New York World's Fair, 1939, New York, Exposition Publications, Incorporated, 1939, p. 118.

\section{RÉSUMÉS}

Dès les premières années de pouvoir de Zog, la volonté de développement du tourisme se mit en place. Comme dans d'autres domaines, les influences étrangères se manifestèrent. Le jeune État et ses sympathisants envisagèrent plusieurs projets, dont certains arrivèrent à terme. Les infrastructures se développèrent et les touristes arrivèrent. Étrangement, aucun véritable travail de synthèse n'a été réalisé sur ce sujet. Afin de combler ce déficit historiographique, nous proposons ici une première description des sources disponibles.

From the early years of Zog's reign power, the will to expand tourism was established. As in other fields, foreign influences appeared. The young state and its sympathizers envisaged several projects, some of which were realized. Infrastructures were developed and tourists appeared. 
Curiously, no real synthesis work has been done on this subject. In order to fill this historiographical gap, we offer here a first description of the available sources.

INDEX

Index géographique: Albanie

Mots-clés : Albanie, Rey (Léon), tourisme, Zog

Keywords : Albania, Rey (Léon), tourism, King Zog

\section{AUTEUR}

\section{PIERRE VILVENS}

Master en histoire à finalité approfondie de l'Université de Liège (2017)

vilvens.pierre[at]gmail.com 